\title{
THE INFLUENCE OF MECHANICAL FACTORS ON THE STRUCTURE OF THE PERIPHERAL ARTERIES AND THE LOCALIZATION OF ATHEROSCLEROSIS
}

\author{
BY \\ J. H. ROBERTSON \\ From the Department of Pathology, Queen's University, Belfast
}

(RECEIVED FOR PUBLICATION DECEMBER 28, 1959)

In a study of the peripheral arteries a period of development lasting until the end of the second decade of life is described. During this time there is evidence of medial hypertrophy and a continued formation of the longitudinal muscular cushions described in the foetus. Bands of longitudinal muscle also appear in the media of certain vessels. It is considered that the findings confirm the functional significance of foetal cushions and further suggest their relationship to the stresses of pulsation. In the later decades the tendency of atherosclerotic plaques to develop at the site of cushions is believed to reflect the importance of mechanical factors in the localization of this disease.

The finding of cushion-like thickenings composed of longitudinal muscle and elastic tissue in the walls of the popliteal and lower end of the brachial artery of the foetus was reported in a previous communication (Robertson, 1960). These occurred mainly at the mouths of branches and were found to be similar to intimal thickenings previously described in the coronary arteries of the foetus (Dock, 1946 ; Minkowski, 1947). It was believed that they represented the physiological reaction of the vessel wall to a pulsatile longitudinal stress, and their presence in only certain of the muscular arteries was attributed to the unrestricted movements of these vessels during pulsation.

Mechanical stresses have been considered by many to be important in the genesis of atherosclerosis, and those due to pulsation have been most often incriminated (Duguid, 1926; Moschcowitz, 1942). For this reason, it was thought that it would be of interest to study the subsequent development of foetal cushions in the peripheral arteries of the adult and to determine their relationship to atherosclerosis. During the course of this work it was observed that many of the changes which occur focally in the arteries of the foetus are continued in a more exaggerated form into early adult life. It is believed that this reflects a continuous process of arterial development which is the physiological response of the vessel to certain well-defined haemodynamic stresses.

\section{Materials}

The peripheral arteries of 81 subjects coming to necropsy were examined, their ages ranging from 1 month to 95 years. All those with clinical or postmortem evidence of hypertension were excluded from the series.

A small segment of each artery studied was taken from the same site in each case. In the upper limb segments were taken from the brachial artery both at the level of the insertion of the deltoid muscle and in the antecubital fossa. In 65 cases, the radial artery at the wrist was examined. In the leg, segments were taken from the femoral artery in the upper part of the subsartorial canal, from the popliteal artery in the popliteal fossa, and from the posterior tibial artery just above the medial malleolus. Sections were stained with haematoxylin and eosin and with Weigert's elastic tissue stain and van Gieson.

The age distribution of the 81 cases is shown in Table I.

TABLE I

AGE DISTRIBUTION

\begin{tabular}{c|c}
\hline Years & No. \\
\hline $0-10$ & 16 \\
$11-20$ & 8 \\
$21-30$ & 8 \\
$31-40$ & 7 \\
$41-50$ & 7 \\
$51-60$ & 9 \\
$61-70$ & 7 \\
$71-80$ & 8 \\
$81-90$ & 8 \\
$91-100$ & 3 \\
\hline
\end{tabular}




\section{Findings}

During the first two decades of life three changes were seen to occur in the structure of the peripheral vessels. The formation of muscular cushions at branch orifices was continued from foetal life and extensive bands of longitudinal muscle developed in the media of the popliteal and lower brachial arteries. In the intima, the internal elastic lamina split into two lamellae to form the musculo-elastic layer.

In the first decade the muscular cushions previously described in the popliteal and lower end of the brachial artery of the foetus showed some increase in size but no other alteration in their appearance. By the end of the second decade, however, cushions had often been formed at the mouths of branches in the other peripheral vessels. They were especially prominent in the femoral artery. Here they frequently rivalled the popliteal cushions in size and were of the same structure, being composed of longitudinal muscle supported by a prominent mesh of elastic tissue (Fig. 1). In the posterior tibial and radial vessels, on the other hand, cushions were much less frequently found and often consisted of only small

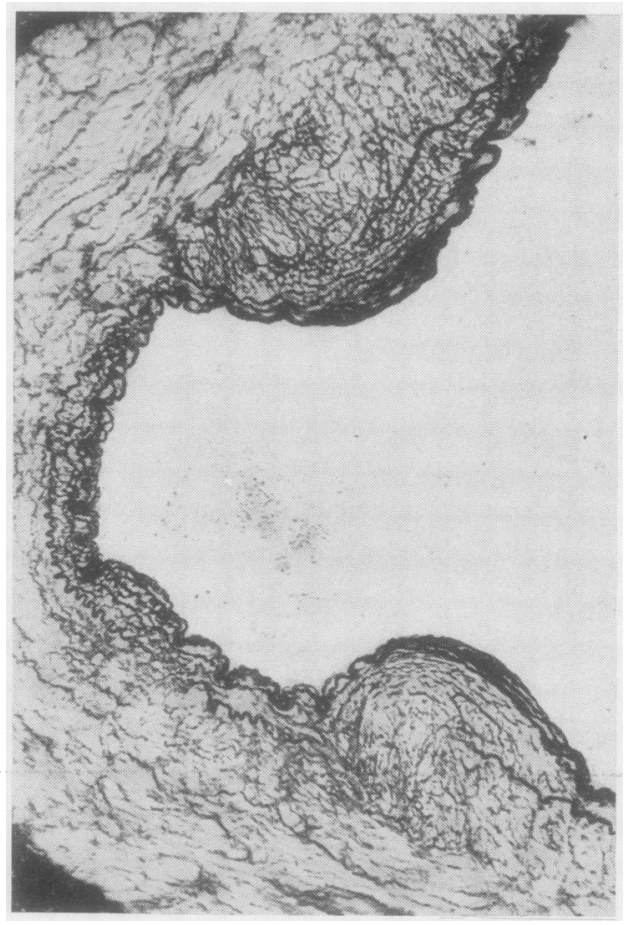

FIG. 1.-Femoral artery. Female, 19 years. Cushions of longitudinal muscle are present on either side of a branch. Weigert $\times 100$. and inconspicuous nodes of longitudinal muscle. Simultaneously with the development of cushions throughout the peripheral vascular system the media of the popliteal and lower brachial arteries began to acquire bands of longitudinal muscle (Fig. 2). This change was not found in any of the other peripheral vessels and was much more marked and constantly present in the popliteal than in the brachial artery. These bands were at first quite narrow, but often extended more than half-way around the circumference of the vessel. During the second decade they became much broader and in some vessels their prominence was quite remarkable, longitudinal muscle bundles forming almost the whole width of the media (Fig. 3).

Towards the end of the first and in the second decade the internal elastica began to split to form the musculo-elastic layer of intima. This occurred fairly uniformly around the circumference of the larger vessels. In the popliteal artery, however, splitting tended to occur first over bands of medial longitudinal muscle and the musculoelastic layer was often thickest in these regions (Figs. 2 and 3). The manner in which the musculo-elastic layer was formed was quite distinctive. Initially, the internal elastica thickened and two component parts of it could be recognized with Weigert's elastic tissue stain. These two lamellae then began to move apart and even in the earliest stages of separation densely staining cell nuclei could be distinguished between them in many places (Fig. 4). As further separation occurred these nuclei could be identified as belonging to smooth muscle cells which were orientated mainly in the longitudinal axis of the vessel. Muscle cells could also be seen to be passing from the media into the newly formed musculo-elastic layer through fenestrae in the internal elastica in a manner similar to that described in the foetus.

At the end of the second and in the later decades the musculo-elastic layer of the intima presented a very varied appearance. In some cases a well-defined layer was present, but in others reduplication of the internal elastica had apparently not yet occurred or was only in its very early stages, even in vessels of the third or fourth decade. The appearances in some sections suggested an explanation for the seemingly tardy formation of this layer. In these, the elastica was duplicated around most of the circumference of the vessel, but in places large gaps had appeared in the outer elastic lamella so that here the musculo-elastic layer had become continuous with 

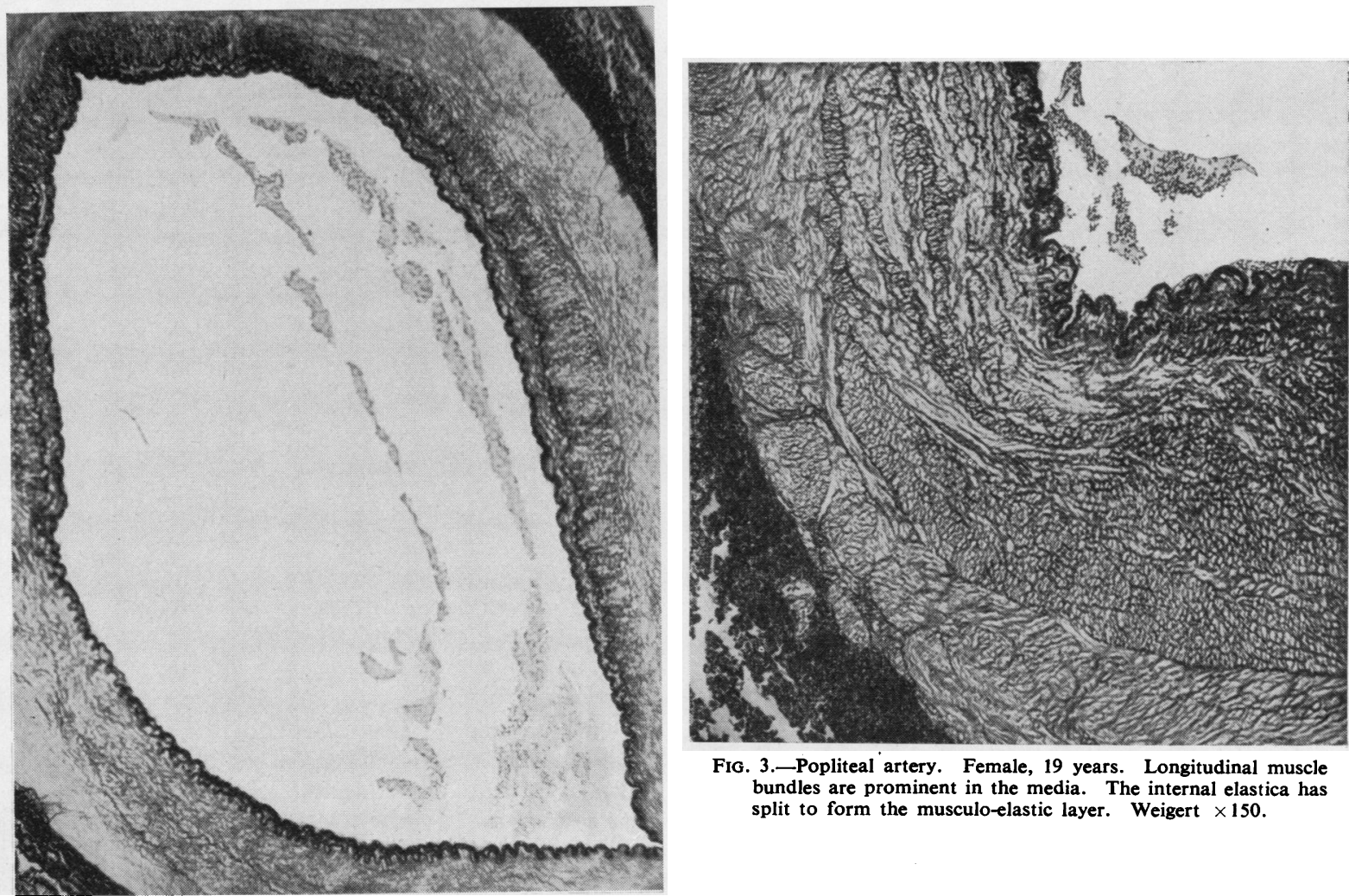

FIG. 3.-Popliteal artery. Female, 19 years. Longitudinal muscle bundles are prominent in the media. The internal elastica has split to form the musculo-elastic layer. Weigert $\times 150$.

FIG. 2-Popliteal artery, Male, 11 years. A band of longitudinal muscle in the media extends around much of the circumference of the vessel. A broad musculo-elastic layer covers it. Weigert $\times 65$.

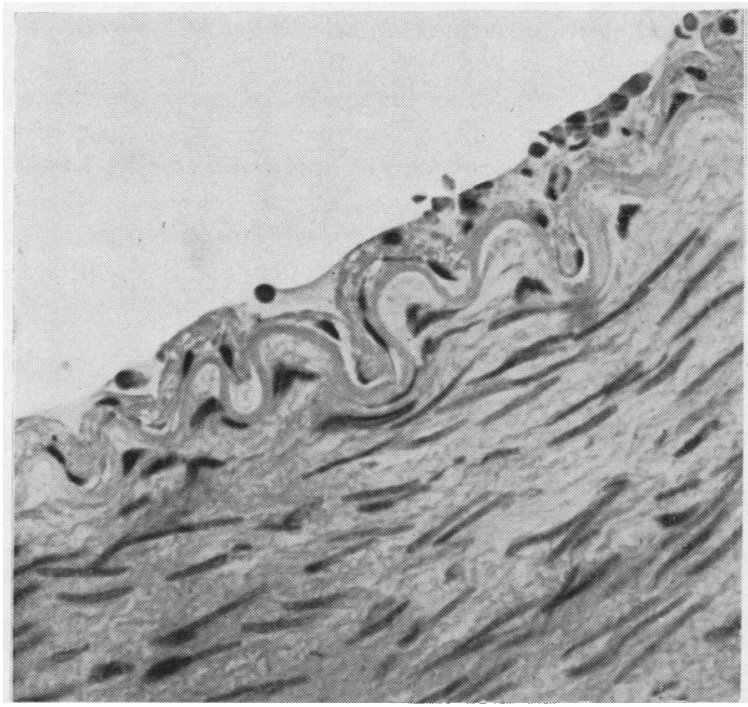

Fig. 4.-An early stage in the formation of the musculo-elastic layer. The internal elastica has begun to split and cell nuclei can be distinguished between its two components. Haematoxylin and $\operatorname{cosin} \times 400$.

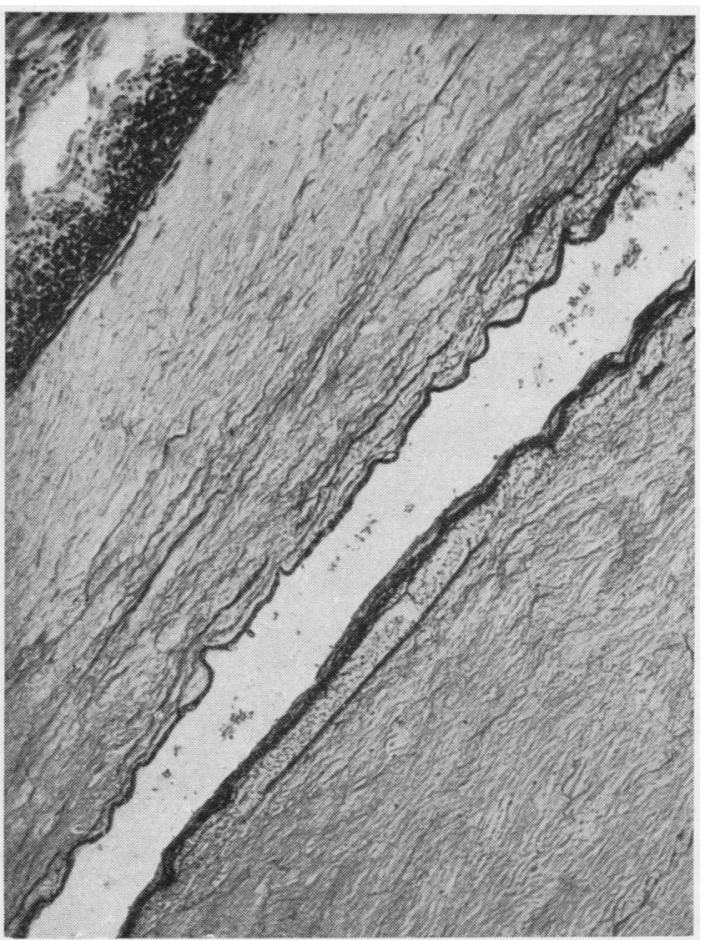

Fig. 5.-Femoral artery. Male, 33 years. Large gaps are present in the outer elastic lamella. In places the elastic stippling of the musculo-elastic layer is qui.e distinct, but in others the media has extended up to the lining elastic membrane. Weigert $\times 8$ ). 


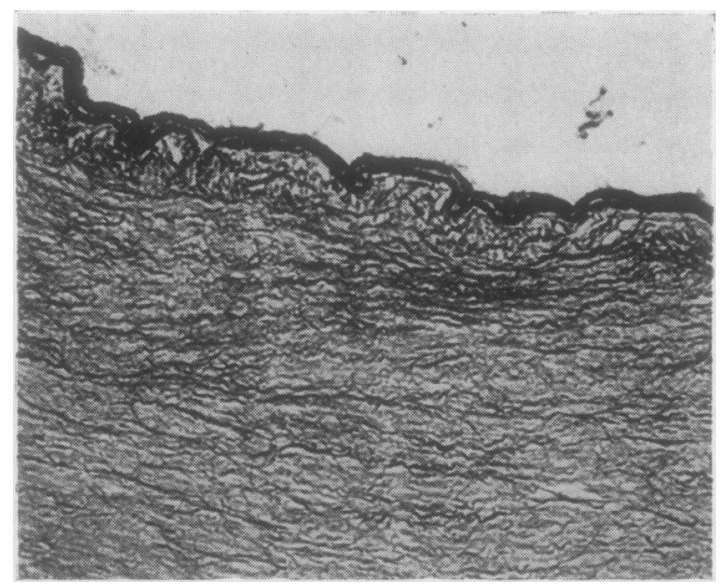

FIG. 6.-The elastic pattern of a former musculo-elastic layer can still be distinguished. Renewed splitting of the internal elastica is occurring. Weigert $\times 220$.

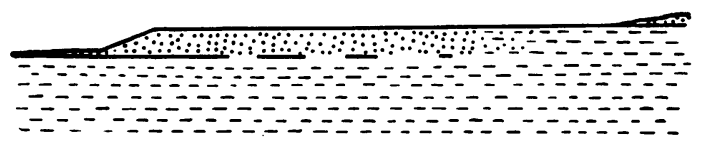

FIG. 7.-From left to right is illustrated the proposed sequence of the formation and incorporation of the musculo-elastic layer into the media. Circular muscle is represented by dashes, longitudinal muscle by dots, and elastic tissue by the continuous lines.

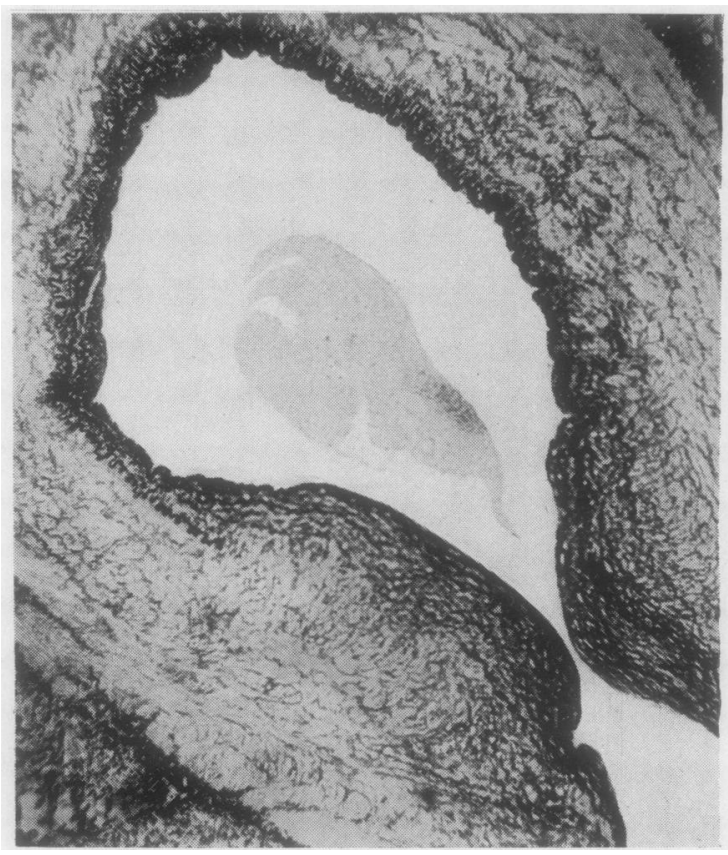

FiG. 8.-Brachial artery. Male, 35 years. Longitudinal muscular cushions are present on either side of a branch. The elastic hyperplastic layer has become thickest over them and already small plaques have been formed. Weigert $\times 70$. the media. In these places the intimal layer could often still be distinguished by virtue of the pattern of its muscle and elastic fibrils, but in parts it had lost its identity and merged with the media (Fig. 5). In other places renewed splitting of the internal elastica was sometimes evident over remnants of a former musculo-elastic layer (Fig. 6). These appearances suggest that the formation of this layer is a repetitive process and that each layer is in turn incorporated into the media as illustrated in Fig. 7.

During the first two decades the intimal changes in the posterior tibial and radial arteries differed from those in the larger trunks described above. Although in some cases a distinct musculo-elastic layer was formed, more often this layer was difficult to distinguish because the internal elastica had split to form several elastic membranes.

The third and later decades were characterized by the development of the elastic hyperplastic layer of the intima and by regressive changes in the muscular cushions and media of the peripheral vessels.

The hyperplastic elastic layer of the intima was formed by the splitting of multiple elastic lamellae from the internal elastica. As the process continued these elastic membranes became progressively finer until there was formed an elastic mesh in the interstices of which there were both smooth muscle cells and connective tissue. During the later decades the elastic hyperplastic layer became thicker and in places nodular. The nodular parts most often occurred over muscular cushions at the mouths of branches and over bands of medial longitudinal muscle (Fig. 8). Accordingly, in the posterior tibial and radial vessels it formed a fairly even layer and became thickest and very uneven in the popliteal artery because of the prominence of longitudinal muscle in this vessel. As the nodular areas increased in size they became vascularized, much more fibrous in character, and lipoid deposits appeared in their substance, completing their transition into atheromatous plaques.

During this period there was a progressive fibrosis of the media with atrophy of its longitudinal muscle. These regressive changes were only gradual in onset and did not become prominent until the fifth decade. Medial fibrosis tended to be most marked beneath developing intimal plaques at the mouths of branches. Here, to a large extent, it replaced the longitudinal muscle of the media so that eventually only remnants of the former muscular cushion could be distinguished beneath the plaque. 


\section{Discussion}

Cushion-like thickenings composed of longitudinal muscle and elastic tissue occur in the walls of the popliteal, lower brachial, and coronary arteries of the foetus. They are found especially at the mouths of branches, and it was considered that their function was to buffer the stresses produced by the anchoring effect of the branch on the systolic elongation of the parent vessel. Their presence in only certain of the muscular arteries was thought to reflect marked pulsation of these vessels due to the lack of support they receive from surrounding tissues (Robertson, 1960).

It is believed that the changes which occur in the peripheral vessels during the first two decades of life emphasize the functional significance of the muscular cushions of the foetus. During this period it appears that the same remodelling process which was begun in the foetus is continued and becomes exaggerated. Muscular cushions continue to be formed and appear in even the small peripheral vessels. Extensive longitudinal muscle bands are developed in the media of the popliteal and, to a lesser extent, the lower brachial arteries. Gross, Epstein, and Kugel (1934) have described how longitudinal muscle also becomes prominent in the media of the coronary arteries by the second decade. The degree of development of these changes can be correlated with the severity of pulsatile stress. In the smaller arteries, where the pulse wave is flattened and less steep (Sodeman, 1956), muscular cushions are small. In those vessels, in which it has been suggested that pulsation is marked, cushions develop earlier, are much larger, and are reinforced by extensive longitudinal muscle bands in the media.

During the first two decades the musculo-elastic layer of the intima is also formed. There have been many interpretations of the significance of this layer. Some have considered it to be a manifestation of growth; others believe it to be degenerative in nature and an inevitable change due to age. MacLean (1929) was of the opinion that any splitting of the internal elastica was pathological as sometimes the coronary arteries of quite elderly subjects showed little evidence of it. Aschoff (1924) regarded the musculo-elastic layer as evidence of hypertrophy in response to a gradual rise in blood pressure. That such a rise does occur with age among the general population has been shown by Master, Dublin, and Marks (1950) and by others.

The results of this study support this last view. It has been described how the formation of this layer is a repetitive process which is primarily medial in origin and results in an increase in the thickness of this coat. Its absence in foetal life argues against it being a manifestation of growth, while its accentuation over medial longitudinal muscle bands tends to associate it with hypertrophy. Wolkoff (1924) has found that in rabbits, cats, dogs, and monkeys the musculo-elastic layer develops only at the margins of branches where also longitudinal hypertrophy is so prominent in the human subject. The manner in which the musculo-elastic layer is formed is also reminiscent of the elastic tissue changes which occur over developing foetal cushions. In both cases smooth muscle cells appear to split the internal elastica mechanically into lamellae which then become increasingly separated by a further ingrowth of medial muscle. In the foetus the outer lamella eventually becomes greatly interrupted and difficult to distinguish, while in the adult it disappears with a resultant increase in the width of the media. It is believed, therefore, that the development and subsequent incorporation of the musculo-elastic layer into the media reflect a hypertrophy of the circular muscle of the vessel in the same way as the foetal cushion represents a local longitudinal hypertrophy.

The repeated formation of the musculo-elastic layer could be expected to have a further result. Not only will it increase the width of the media but there will also be a reduction in the radius of the lumen of the vessel. According to Burton (1951), the law of Laplace applies to blood vessels. This states that the tension developed in the wall (T) is proportional not only to the excess of hydrostatic pressure in the lumen over the local surrounding tissue pressure $(\mathrm{P})$ but also to the radius of the lumen ( $R)$, that is, $T=P \times R$. Thus, the repeated formation of the musculo-elastic layer constitutes a physiologically efficient method of compensating for a rise in blood pressure. Not only is the muscular bulk of the wall increased but also the radius of the lumen is decreased, so tending to reduce the tension in the wall and the need for hypertrophy.

The prominent part played by medial smooth muscle in the formation of the musculo-elastic layer has been described. This supports the observations of previous authors. Jores (1903, quoted by McMeans, 1915) believed that splitting of elastic tissue is accomplished mechanically by smooth muscle. Gross et al. (1934) and Altschul (1950) have both described the passage of smooth muscle from the media into the intima. Altschul described it in both human and experimental arteriosclerosis and considered that vascular 
smooth muscle may possibly be capable of amoeboid movements. More recently, Morgan (1956) has confirmed that many of the smooth muscle cells of atherosclerotic plaques are derived from ingrowths of medial muscle.

During the first two decades the musculoelastic layer of the posterior tibial and radial arteries was difficult to distinguish because of the frequent formation of several elastic lamellae between which were many smooth muscle cells. It seems possible that this change represents an exaggerated form of the musculo-elastic layer. Mitchell (1956) has observed that the smaller peripheral arteries have a more profuse vascular innervation and relatively thicker muscular coats than the more proximal trunks. If active vasomotor function requires these vessels to have an unusually thick muscular coat it could be expected that a rise in blood pressure would result in a more urgent stimulus to compensatory processes than in the larger vessels. It is possible, therefore, that successive musculo-elastic layers are formed more rapidly in the smaller vessels and that the rate of reduplication of elastic membranes exceeds the rate at which they degenerate and disappear.

In the larger vessels the development of the elastic hyperplastic layer is associated with the onset of medial fibrosis and the period of decline in arterial development. Many believe that the formation of this layer is secondary to degenerative changes in the underlying media (Thoma, 1883 ; Blumenthal, Lansing, and Gray, 1950 ; Crawford and Levene, 1953). The present study would support this view. Medial fibrosis was found to be most marked in those areas of longitudinal hypertrophy thought to be subject to haemodynamic stress. Over these regions the elastic hyperplastic layer tended to become nodular and, in the later decades, atheromatous. In this way, many foetal cushions mark the site of a future atherosclerotic plaque, and, as Morgan (1956) has shown, atherosclerosis becomes common in the popliteal as well as in the coronary arteries.

The influence of pulsatile stresses in causing the localization of atherosclerosis at the mouths of branches has been stressed by Duguid (1926). It is believed that the muscular cushions of the foetus and the later development of the peripheral arteries traced in this study confirm the presence of such a stress and indicate its importance in the localization of intimal plaques.

I wish to thank Professor J. H. Biggart for his help and advice during the course of this work.

\section{REFERENCES}

Altschul, R. (1950). Selected Studies on Arteriosclerosis, p. 50 . Z Thomas, Springfield, Illinois.

Aschoff, L. (1924). Lectures on Pathology, pp. 131-153. Hoeber, New York.

Blumenthal, H. T., Lansing, A. I., and Gray, S. H. (1950). Amer. O J. Path., 26, 989.

Burton, A. C. (1951). Amer. J. Physiol., 164, 319.

Crawford, T., and Levene, C. I. (1953). J. Path. Bact., 66, 19.

Dock, W. (1946). J. Amer. med. Ass., 131, 875.

Duguid, J. B. (1926). J. Path. Bact., 29, 371.

Gross, L., Epstein, E. Z., and Kugel, M. A. (1934). Amer. J. Path. $10,253$.

MacLean, D. L. (1929). Ann. intern. Med., 2, 1253.

McMeans, J. W. (1915). J. med. Res., 32, 377.

Master, A. M., Dublin, L. I., and Marks, H. H. (1950). J. Amer. med. Ass., 143, 1464.

Minkowski, W. L. (1947). Amer. J. med. Sci., $214,623$.

Mitchell, G. A. G. (1956). Cardiovascular Innervation, p. 70 . Livingstone, Edinburgh and London.

Morgan, A. D. (1956). The Pathogenesis of Coronary Occlusion. Blackwell, Oxford.

Moschcowitz, E. (1942). Vascular Sclerosis, p. 129. Oxford University Press, New York.

Robertson, J. H. (1960). J. clin. Path., 13, 133.

Sodeman, W. A. (1956). Pathologic Physiology, 2nd ed. Saunders, London and Philadelphia.

Thoma, R. (1883). Virchows Arch. path. Anat., 93, 443.

Wolkoff, K. (1924). Ibid., 252, 208. 